\title{
Long-Term Evaluation of Combined Antihypertensive Therapy with Lisinopril and a Thiazide Diuretic in Patients with Essential Hypertension
}

\author{
Toshihiko IsHImITSU, ${ }^{1} \mathrm{MD}$, Shigeru YAGI,${ }^{1} \mathrm{MD}$, \\ Akio Ebihara, ${ }^{2} \mathrm{MD}$, Yutaka Dol, ${ }^{3} \mathrm{MD}$, \\ Akira DOMAE, ${ }^{4} \mathrm{MD}$, Akira Shibata, ${ }^{5} \mathrm{MD}$, \\ Masayuki Kimura, ${ }^{6} \mathrm{PhD}$, Yasuro Sugishita, ${ }^{7} \mathrm{MD}$, \\ Etsuro Sagara, ${ }^{8} \mathrm{PhD}$, Tetsuo Sakamaki, ${ }^{9} \mathrm{MD}$, \\ and Kazuhiko Murata, ${ }^{9} \mathrm{MD}$
}

\begin{abstract}
SUMMARY
For the treatment of hypertension, the combination of an angiotensinconverting enzyme (ACE) inhibitor and a thiazide diuretic is supported by multiple lines of evidence, because these drugs have synergistic action and are expected to cancel out each other's adverse side effects. However, the longterm outcome of this combination antihypertensive therapy is not entirely clear. In the present multicenter open trial, we investigated the long-term efficacy and safety of combined antihypertensive therapy with an ACE inhibitor, lisinopril, and a thiazide diuretic, trichlormethiazide. A total of 466 patients with essential hypertension were treated with lisinopril alonc (monotherapy group, $n=360$ ) or with a combination of lisinopril with trichlormethiazide (combination therapy group, $n=106$ ) for 1 year. The average blood pressure was effectively lowered to below $150 / 90 \mathrm{mmHg}$ in both the monotherapy and the combination therapy groups throughout the study period. The average maintenance dose of lisinopril was lower when combined with thiazide than when given alone $(9.8$ vs. $11.5 \mathrm{mg} /$ day, $p<0.001)$. Dry cough was the major side effect of lisinopril; no severe adverse effects were observed. The incidence of cough was not significantly different between the monotherapy group (13.1\%) and the combination therapy group $(11.3 \%)$. The increase in serum potassium observed in the monotherapy group was reversed by the concurrent use of the thiazide
\end{abstract}

From the 'Department of Medicine, Division of Hypertension and Cardiorenal Diseases, Dokkyo University School of Medicine, Tochigi, Japan, ${ }^{2}$ Department of Clinical Pharmacology, Jichi Medical School, Tochigi, Japan, ${ }^{3}$ Second Department of Internal Medicine, Saitama Medical School, Saitama, Japan, ${ }^{4}$ Health Administration Center, Saitama University, Urawa, Japan, ${ }^{5}$ First Department of Internal Medicine, Niigata University School of Medicine, Niigata, Japan, ${ }^{6}$ Department of Pharmacy Services, Saitama Medical Center, Saitama Medical School, Kawagoe, Japan, ${ }^{7}$ Department of Medicine, Cardiovascular Division, and ${ }^{8}$ Department of Clinical Pharmacy, University of Tsukuba, Tsukuba, Japan, ${ }^{9}$ Second Department of Internal Medicine, Gunma University School of Medicine, Maebashi, Japan.

Address for correspondence: Toshihiko Ishimitsu, MD, Department of Medicine, Division of Hypertension and Cardiorenal Diseases, Dokkyo University School of Medicine, Mibu, Tochigi 321-02, Japan.

Received for publication January 22, 1997.

Accepted July 23, 1997. 
diuretic in the combination therapy group. Fasting blood glucose was significantly reduced in the monotherapy group; the reduction observed in the combination therapy group was not significant. Thus, the present results provide useful information as to the effectiveness and safety of combined antihypertensive therapy with lisinopril and a thiazide in comparison with monotherapy with lisinopril. (Jpn Heart J 1997; 38: 831-840)

Key words: Angiotensin-converting enzyme inhibitors, Thiazide diuretics, Hypertension, Cough

7 HE development of new antihypertensive drugs with various mechanisms of action has greatly facilitated the control of blood pressure in hypertensive patients. Therefore, in treating hypertension, more attention should now be directed towards preventing organ damage such as arteriosclerosis and cardiac hypertrophy in an efficient manner, thereby reducing the incidence of cerebrovascular diseases, coronary heart disease, and renal failure. However, the results of epidemiological studies ${ }^{1-3)}$ have raised the possibility that traditional antihypertensive therapy with diuretics and $\beta$-blockers may not be effective in preventing coronary heart disease, although it substantially reduces the incidence of cerebrovascular diseases. Diuretics and $\beta$-blockers adversely affect lipid and glucose metabolism, which may actually increase the risk of coronary heart disease. ${ }^{4-6)}$ Angiotensin-converting enzyme (ACE) inhibitors do not have adverse effects on serum lipids, and have been shown to improve glucose metabolism by increasing the sensitivity of hypertensive patients to insulin. ${ }^{7-10)}$

The combination of an ACE inhibitor and a thiazide diuretic is recommended by multiple lines of evidence, not only because they are synergistic in terms of mode of action but also because they are expected to cancel out each other's adverse side effects. ${ }^{11,12)}$ However, the long-term outcome of interactions between ACE inhibitors and thiazides has not been studied in detail. In this multicenter open trial, we investigated the long-term efficacy and safety of this combination therapy in comparison with ACE inhibitor monotherapy in hypertensive patients.

\section{Subjects and Methods}

An open-label clinical trial was performed at 7 university hospitals and 90 related hospitals located to the north of Tokyo. Patients with essential hypertension, treated or previously untreated, 30 to 80 years of age, were enrolled in the trial. Informed consent was obtained from all subjects. The study protocol was in accordance with the declaration of Helsinki (1989) of the World Medical Association. The exclusion criteria were 1) severe brain, heart or kidney damage; 2) the presence of other severe diseases, such as liver failure or malignant tumors; 3) the 
presence of a secondary cause of hypertension; 4) the presence of chronic diseases such as diabetes mellitus; and 5) other conditions judged by the attending physician to contraindicate the use of the study drugs, such as pregnancy or hypersensitivity.

Blood pressure was measured by a physician using a mercury manometer with the patient in the sitting position. Untreated patients or patients treated with the thiazide diuretic, trichlormethiazide, alone were eligible if their systolic pressure was higher than $160 \mathrm{mmHg}$, their diastolic pressure was higher than 95 $\mathrm{mmHg}$, or both, during a 4-week run-in period. Treatment with lisinopril was begun at a dose of $10 \mathrm{mg}$ for untreated patients (the monotherapy group, $M$ ) and 5 or $10 \mathrm{mg}$ for patients already treated with trichlormethiazide (the combination therapy group, C) once daily, orally. During subsequent visits, the dose of lisinopril was titrated within the range of 5 to $20 \mathrm{mg}$ per day, so that the sitting blood pressure was maintained under $150 / 90 \mathrm{mmHg}$. The patients were treated with lisinopril for up to 12 months. The average dose of trichlormethiazide was $2.1 \pm 0.4 \mathrm{mg} /$ day and the dose was not changed during the study period. The patients were withdrawn from the study in the following cases: 1) the development of severe adverse reactions or worsening of symptoms; 2) failure to control blood pressure, even with the maximum dose of lisinopril, and the necessity for additional antihypertensive medication; 3) poor compliance with treatment; 4) the development of severe concomitant diseases.

Besides the monitoring of blood pressure, pulse rate, and clinical condition at each visit, laboratory tests were performed before treatment and 12 months after the initiation of treatment. The laboratory tests included urinalysis, blood cell counts, fasting blood total protein, total cholesterol, high-density lipoprotein (HDL) cholesterol, triglycerides, aspartate aminotransferase, alanine aminotransferase, alkaline phosphatase, lactate dehydrogenase, $\gamma$-glutamyl transpeptidase, creatine phosphokinase, blood urea nitrogen, creatinine, uric acid, sodium, potassium, chloride, and glucose. Aspartate aminotransferase and alanine aminotransferase are expressed in Karmen units $(\mathrm{KU})$, and alkaline phosphatase, lactate dehydrogenase, $\gamma$-glutamyl transpeptidase and creatine phosphokinase in international units (IU). Blood samples were drawn from an antecubital vein in the morning after an overnight fast.

Data are presented as the mean \pm SD. Time-course changes in variables were evaluated by analysis of variance for repeated measures. A $p$ valuc less than 0.05 was considered to indicate statistical significance.

\section{Results}

A total of 514 patients were initially registered in the study, however, 48 
were not included because of failure to meet the inclusion criteria or violation of the study protocol. Thus, 466 patients, 360 in the $\mathrm{M}$ group and 106 in the $\mathrm{C}$ group, met the inclusion criteria and were enrolled. Of these patients, 11 (M9, C2) were transferred to other hospitals mainly because of changing the place of residence. Nine patients $(M 8, G 1)$ decided not to visit the hospital due to personal reasons; however, follow-up inquiry by telephone revealed that they were doing well. The administration of lisinopril was stopped in 7 patients (M5, C2) because the hypotensive effect was excessive. Conversely, in 9 patients (M6, C3), addi-

Table I. Baseline Characteristics of the 354 Patients

\begin{tabular}{lcc}
\hline \multicolumn{1}{c}{ Variable } & Monotherapy group $(\mathbf{M})$ & Combination therapy group $(\mathrm{C})$ \\
\hline Age $(\mathrm{yr})$ & $58 \pm 12$ & $59 \pm 11$ \\
Sex (male / female) & $147 / 127$ & $35 / 45$ \\
Body Mass Index $\left(\mathrm{kg} / \mathrm{m}^{2}\right)$ & $23.9 \pm 3.3$ & $23.9 \pm 3.7$ \\
Duration of hypertension $(\mathrm{yr})$ & $5 \pm 5$ & $6 \pm 6$ \\
Systolic blood pressure $(\mathrm{mmHg})$ & $170 \pm 18$ & $171 \pm 18$ \\
Diastolic blood pressure $(\mathrm{mmHg})$ & $98 \pm 11$ & $100 \pm 10$ \\
Pulse rate (beats/min) & $76 \pm 12$ & $76 \pm 10$ \\
WHO stage I & 187 & 55 \\
$\quad$ II & 80 & 24 \\
\multicolumn{1}{l}{ III } & 7 & 1 \\
\hline
\end{tabular}

Data are mean $\pm \mathrm{SD}$.
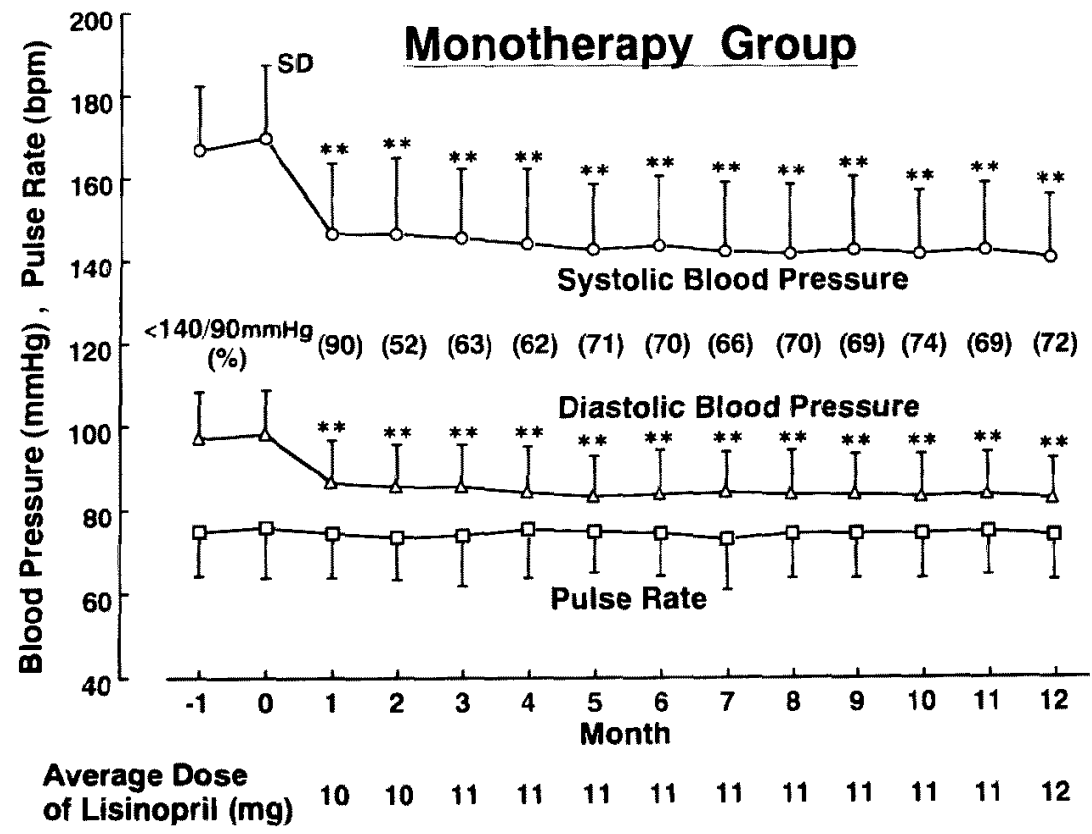

Figure 1. Time-course changes in blood pressure, pulse rate, and dose of lisinopril during the study period in the 360 hypertensive patients of the monotherapy group. ${ }^{* *} p<0.01$ vs. the baseline value. Numbers in parentheses indicate percentages of patients showing normotensive range blood pressure $(<140 / 90 \mathrm{mmHg})$. 


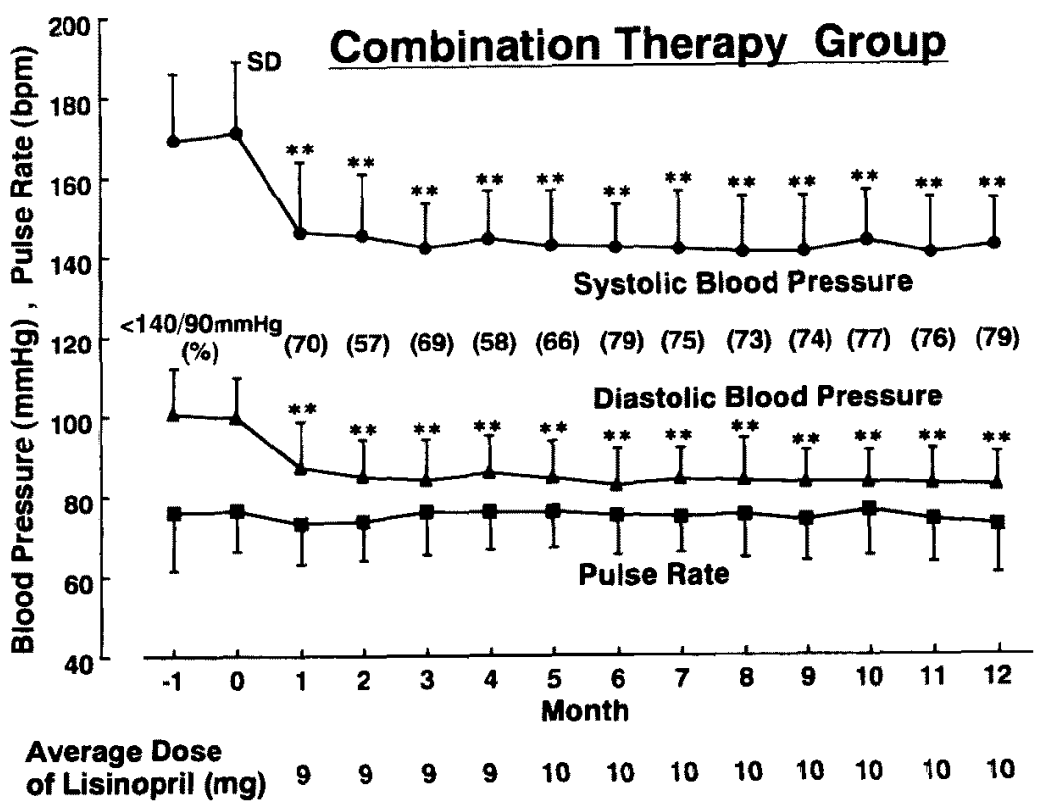

Figure 2. Time-course changes in blood pressure, pulse rate, and dose of lisinopril during the study period in the 106 hypertensive patients of the combination therapy group. ${ }^{* *} p<0.01$ vs. the baseline value. Numbers in parentheses indicate percentages of patients showing normotensive range blood pressure $(<140 / 90 \mathrm{mmHg})$.

Table II. Blood Cell Counts and Body Weight Before and After the Study Period

\begin{tabular}{|c|c|c|c|c|}
\hline \multirow{2}{*}{ Variable } & \multicolumn{2}{|c|}{ Monotherapy group (M) } & \multicolumn{2}{|c|}{ Combination therapy group $(\mathrm{C}$} \\
\hline & Before & After & Before & After \\
\hline White blood cells $\left(\times 10^{3} / \mathrm{mm}^{3}\right)$ & $6.3 \pm 1.5$ & $6.2 \pm 1.4$ & $6.3 \pm 1.6$ & $6.4 \pm 1.4$ \\
\hline Red blood cells $\left(\times 10^{6} / \mathrm{mm}^{3}\right)$ & $4.62 \pm 0.49$ & $4.46 \pm 0.53^{\#}$ & $4.67 \pm 0.48$ & $4.57 \pm 0.43 * *$ \\
\hline Hemoglobin concentration $(\mathrm{g} / \mathrm{L})$ & $142 \pm 16$ & $138 \pm 17^{\#}$ & $141 \pm 16$ & $139 \pm 14 *$ \\
\hline Hematocrit (\%) & $42.8 \pm 4.4$ & $41.4 \pm 4.9^{\#}$ & $42.9 \pm 4.4$ & $42.1 \pm 4.2^{* *}$ \\
\hline Platelets $\left(\times 10^{3} / \mathrm{mm}^{3}\right)$ & $226 \pm 52$ & $224 \pm 48$ & $249 \pm 59$ & $251 \pm 49$ \\
\hline Body weight $(\mathrm{kg})$ & $58.9 \pm 12.4$ & $58.5 \pm 12.5$ & $60.0 \pm 10.9$ & $60.5 \pm 10.8$ \\
\hline
\end{tabular}

Data are mean \pm SD. ${ }^{*} p<0.05, * * p<0.01,{ }^{*} p<0.001$ vs. values before treatment.

tional antihypertensive medication was needed to control blood pressure. Lisinopril was discontinued in 61 patients because of side effects; in most cases, dry cough was the cause for discontinuation (M47, G12). The incidence of cough was insignificantly lower in the $\mathrm{C}$ group than in the $\mathrm{M}$ group (11.3\% vs. 13.1\%). Two patients in the $\mathrm{C}$ group developed allergic dermatitis and lisinopril was stopped. The study protocol was abandoned in 1 patient in the $\mathrm{C}$ group because of pneumonia and in 2 patients in the $M$ group because of aggravation of concomitant diseases (gastric ulcer and ventricular arrhythmia). Twelve (M9, C3) patients were excluded from the data analysis because of poor compliance. 
The data were analyzed in the remaining 354 patients (M274, C80). Table I lists the baseline characteristics of these patients in each group. The two groups had comparable values in terms of age, sex ratio, body mass index, and history of hypertension. Although the patients in $\mathrm{C}$ group had been already treated with trichlormethiazide, their blood pressure was in the hypertensive range and either the systolic or the diastolic values were on a par with those of the M group. Pulse rate also did not differ between the two groups. The degree of hypertensive organ damage was comparable in the two groups when classified according to the WHO criteria. Four patients (M3, G1) had a past history of myocardial infarction, 3 patients in the $M$ group had had a cerebral infarction in the past, and I patient in the $\mathbf{M}$ group showed an exudative lesion in the retina. They were classified as WHO III; however, the sequent organ damage was not severe and they were judged to be eligible for the study protocol.

Figures 1 and 2 show the time-course changes in blood pressure and pulse rate during the study period in the $\mathrm{M}$ and the $\mathrm{C}$ groups, respectively. In both groups, the average blood pressure was effectively lowered to below $150 / 90$ $\mathrm{mmHg}$ one month after the start of lisinopril treatment. This antihypertensive effect was well maintained throughout the study period. The average maintenance dose was significantly lower in the $\mathrm{C}$ group than in the $\mathrm{M}$ group $(9.8 \pm 3.9$ vs. $11.5 \pm 4.2 \mathrm{mg} /$ day, $p<0.001$ ). No changes in pulse rate were observed during the study period in either group.

The time-course changes in blood cell counts and body weight are shown in

Table III. Blood Chemistry before and after the Study Period

\begin{tabular}{|c|c|c|c|c|}
\hline \multirow{2}{*}{ Variable } & \multicolumn{2}{|c|}{ Monotherapy group $(\mathrm{M})$} & \multicolumn{2}{|c|}{ Combination therapy group $(\mathrm{C})$} \\
\hline & Before & After & Before & After \\
\hline Total protein $(g / l)$ & $72 \pm 5$ & $71 \pm 7$ & $73 \pm 4$ & $71 \pm 5$ \\
\hline Total cholesterol $(\mathrm{mmol} / \mathrm{l})$ & $5.31 \pm 1.03$ & $5.13 \pm 0.92$ & $5.11 \pm 0.90$ & $5.08 \pm 0.87$ \\
\hline HDL-cholesterol $(\mathrm{mmol} / \mathrm{t})$ & $1.33 \pm 0.40$ & $1.32 \pm 0.38$ & $1.38 \pm 0.52$ & $1.36 \pm 0.42$ \\
\hline Triglycerides (mmol/l) & $1.56 \pm 1.11$ & $1.65 \pm 1.14$ & $1.56 \pm 1.11$ & $1.65 \pm 1.14$ \\
\hline Aspartate aminotransferase (KU/l) & $25 \pm 9$ & $25 \pm 13$ & $28 \pm 21$ & $28 \pm 25$ \\
\hline Alanine aminotransferase $(\mathrm{KU} / \mathrm{l})$ & $23 \pm 13$ & $23 \pm 16$ & $23 \pm 15$ & $22 \pm 16$ \\
\hline Alkaline phosphatase (IU/l) & $163 \pm 57$ & $145 \pm 53^{*}$ & $169 \pm 95$ & $168 \pm 82$ \\
\hline Lactate dehydrogenase (IU/l) & $346 \pm 81$ & $345 \pm 81$ & $370 \pm 102$ & $366 \pm 99$ \\
\hline$\gamma$-glutamyl transpeptidase $(\mathrm{IU} / l)$ & $34 \pm 38$ & $39 \pm 61$ & $52 \pm 126$ & $46 \pm 103$ \\
\hline Creatine phosphokinase (IU/l) & $95 \pm 54$ & $95 \pm 55$ & $106 \pm 66$ & $117 \pm 91$ \\
\hline Blood urea nitrogen $(\mathrm{mmol} / \mathrm{l})$ & $11 \pm 3$ & $12 \pm 4$ & $11 \pm 2$ & $12 \pm 2$ \\
\hline Creatinine $(\mu \mathrm{mol} / l)$ & $78 \pm 22$ & $81 \pm 29$ & $68 \pm 19$ & $69 \pm 19$ \\
\hline Uric acid $\left(\mu_{\mathrm{mnol}} / l\right)$ & $315 \pm 88$ & $324 \pm 89$ & $308 \pm 68$ & $305 \pm 67$ \\
\hline Sodium $(\mathrm{mEq} / l)$ & $141 \pm 3$ & $141 \pm 2$ & $141 \pm 2$ & $141 \pm 3$ \\
\hline Potassium $(\mathrm{mEq} / \mathrm{l})$ & $4.1 \pm 0.4$ & $4.3 \pm 0.5^{*}$ & $4.1 \pm 0.4$ & $4.2 \pm 0.4$ \\
\hline Chloride ( $\mathrm{mEq} / l)$ & $103 \pm 3$ & $103 \pm 3$ & $103 \pm 3$ & $103 \pm 3$ \\
\hline Glucose (mmol/t) & $6.5 \pm 2.5$ & $6.0 \pm 2.1 * *$ & $6.1 \pm 2.6$ & $5.8 \pm 1.1$ \\
\hline
\end{tabular}

Data are mean $\pm \mathrm{SD} . * p<0.05, * * p<0.01$ vs. values before treatment. 
Table II. Red blood cells were slightly but significantly reduced after treatment in both groups; however, the values remained within the normal range and lisinopril was not withdrawn from any patient because of this hematological effect. White blood cells and platelets were not affected by the 12-month lisinopril treatment. Body weight did not change significantly during the study period.

Table III presents the blood chemistry data before and after lisinopril treatment. There were no significant changes in liver or renal function, or serum protein and lipids. With regard to the electrolytes, the serum potassium was significantly increased by lisinopril treatment in the $\mathrm{M}$ group but not in the $\mathrm{C}$ group. Fasting blood glucose was significantly reduced after lisinopril treatment in the $\mathrm{M}$ group; the reduction observed in the $\mathrm{C}$ group was not significant.

\section{Discussion}

In the present study, long-term treatment with lisinopril, either alone or in combination with a thiazide diuretic, was effective in controlling the blood pressure of patients with essential hypertension. Moreover, the data suggest that the concurrent use of a thiazide diuretic can reduce the maintenance dose of lisinopril. The major adverse effect of the lisinopril therapy was dry cough, as is the case with ACE inhibitors. ${ }^{13)}$ Although this is not a severe side effect, a total of $12.6 \%$ patients stopped taking lisinopril because of this side effect. The incidence of cough induced by lisinopril in this study is comparable to that seen with other ACE inhibitors. ${ }^{13)}$ Although the mechanism of cough induced by ACE inhibitors is not fully understood, it has been speculated that a buildup of ACE substrates other than angiotensin I, such as bradykinin and substance $P$, may be involved. ${ }^{14}$ Thiazide diuretics have been shown to potentiate acetylcholine-induced airway constriction. ${ }^{15)}$ It has also been reported that thiazides, when aerosolized and inhaled, inhibited a capsaicin-induced increase in airway resistance and cough. ${ }^{16}$ Therefore, it is possible that the addition of thiazides affects the incidence of cough in patients treated with ACE inhibitors. In the present study, the incidence of cough induced by lisinopril did not differ between the monotherapy group and the combination therapy group. McEwan et al. reported that the concurrent use of cyclooxygenase inhibitors attenuates the ACE inhibitor-induced cough. ${ }^{17)}$ However, the long-term use of nonsteroidal antiinflammatory drugs is questionable because it may cause other adverse effects. Thus, the occurrence of cough seems an intractable drawback of ACE inhibitors, although it is not a severe side effect.

Hyperkalemia is another possible side effect of ACE inhibitors. Angiotensin II, a stimulator of aldosterone production, is reduced by ACE inhibitors. Because aldosterone promotes potassium excretion in exchange for sodium reabsorption 
in the distal tubules of kidney, a decrease in aldosterone results in the retention of potassium. In the present study, a significant increase in serum potassium was also observed after a year of lisinopril therapy in the monotherapy group. However, the lisinopril therapy was not stopped in any patient because of hyperkalemia during the study period. This increase in serum potassium was not significant in the combination therapy group. Contrary to the effect of ACE inhibitors, thiazide diuretics facilitate the excretion of potassium along with sodium. Therefore, it is surmised that the effect of lisinopril on potassium balance was compensated for by the counteraction of trichlormethiazide in the combination therapy group of this study. The combination of an ACE inhibitor and a thiazide diuretic thus scems preferable in terms of preventing serum potassium alterations.

Slight but significant decreases in red cell numbers were observed in both the $\mathrm{M}$ and $\mathrm{C}$ groups, although bleeding disorders wcre not obscrved during the study period, except in a patient who suffered an aggravation of gastric ulcer and eventually was not included in the data analysis. It has been reported that ACE inhibitors such as captopril can cause anemia especially in hemodialysis patients. ${ }^{18)}$ A sulfhydril radical of the captopril molecule was supposed to play a role in this side effect; however, later studies have revealed that the ACE inhibitors lacking sulfhydril radicals, such as enalapril, can also cause anemia. ${ }^{19}$ ) Lisinopril used in this study also lacks the sulfhydril radical. Another possible mechanism for the ACE inhibitor-induced anemia is a suppression of erythropoietin production. ${ }^{20)}$ Indeed, ACE inhibitors have been shown to suppress excessive erythropoiesis after renal transplantation. ${ }^{21)}$ Because none of the patients in this study were forced to stop taking lisinopril by anemia, this hematological side effect is not likely to limit the use of lisinopril in hypertensive patients without renal failure.

It is generally thought that ACE inhibitors do not affect the plasma lipid profile. ${ }^{7,8)}$ Some studies have reported reductions in plasma triglycerides by ACE inhibitors ${ }^{22)}$; however, the effect was not consistent in other studies, ${ }^{7,23)}$ and the changes in triglycerides may be related to the improvement of insulin sensitivity. In the monotherapy group of the present study, neither the cholesterol level nor that of triglycerides was affected by chronic lisinopril treatment. Diuretics, however, are known to increase serum LDL-cholesterol and triglycerides, ${ }^{4,5)}$ although it is unclear whether elevated levels persist after years of treatment. ${ }^{24,25)}$ It is of interest that ACE inhibitors have been reported to blunt this untoward effect of diuretics on lipid metabolism. ${ }^{12)}$ However, in the combination group of the present study, no changes in serum lipids were observed after 1 year of concurrent lisinopril administration. Therefore, it is surmised that the effect of ACE inhibitors on lipid metabolism is small, if any, in the chronic treatment of hyper- 
tension.

In summary, the results of the present study show that long-term antihypertensive treatment with an ACE inhibitor, lisinopril, was effective in controlling blood pressure both when given as monotherapy and when combined with a thiazide diuretic. Dry cough was the major side effect of lisinopril; no severe adverse effects were observed. The concurrent use of a thiazide diuretic reduced the maintenance dose of lisinopril and canceled the increase in serum potassium. However, the incidence of cough was not significantly different between the monotherapy and the combination therapy groups. The effects of lisinopril on glucose and lipid metabolism were small, if any, in the long-term treatment of hypertension. Thus, the present data delineate the effectiveness and safety of combination antihypertensive therapy with lisinopril and a thiazide in comparison with monotherapy with lisinopril.

\section{ACKNoWLedgment}

We thank all the attending physicians who participated in this study. This study was supported by a grant for medical research from Shionogi \& Co., Ltd.

\section{REFERENCES}

1. Helgeland A. Treatment of mild hypertension: a five-year controlled drug trial: The Oslo Study. Am J Med 1980; 69: 725-32.

2. Multiple Risk Factor Intervention Trial Research Group. Multiple risk factor intervention trial. JAMA 1982; 248: 1465-77.

3. Medical Research Council Working Party. MRC trial of treatment of mild hypertension: principal results. Br Med J 1985; 291: 97-102.

4. Weidman P, de Courten M, Ferrari P, Böhlen I. Serum lipoproteins during treatment with antihypertensive drugs. J Cardiovasc Pharmacol 1993; 22 (Suppl 6): S98-S105.

5. Black HR. Metabolic consideration in the choice of therapy for the patient with hypertension. Am Heart J 1991; 121: 707-15.

6. Gambardella S, Frontini S, Pellegrinotti M, Testa G, Spallone V, Menzinger G. Carbohydrate metabolism in hypertension: influence of treatment. J Cardiovasc Pharmacol 1993; 22 (Suppl 6): S87-S97.

7. Malini PL, Strochi E, Ambrosioni E, Magnani B. Long-term antihypertensive, metabolic and cellular cffects of enalapril. J Hypertens 1984; 2 (Suppl 2): 101-5.

8. Leren P, Foss PO, Nordvic B, Fossbakk B. The effect of enalapril and timolol on blood lipids: a randomized multicenter hypertension study in general practice in Norway. Acta Med Scand 1988; 223: $321-6$.

9. Pollare $\mathrm{T}$, Lithell $\mathrm{H}$, Berne $\mathrm{C}$. A comparison of the effects of hydrochlorothiazide and captopril on glucose and lipid metabolism in patients with hypertension. N Engl J Med 1989; 321: 868-73.

10. Berne C, Pollare $T$, Lithell $H$. Effects of antihypertensive treatment on insulin sensitivity with special reference to ACE inhibitors. Diabetes Care 1991; 14 (Suppl 4): 39-47.

11. Tobian L, Brunner HR, Cohn JN, ct al. Modern strategies to prevent coronary sequelae and stroke in hypertensive patients differ from the JNC V consensus guidelines. Am J Hypertens 1994; 7: 859-72.

12. Weinberger $\mathrm{MH}$. Influence of an angiotensin converting-enzyme inhibitor on diuretic-induced metabolic effects in hypertension. Hypertension 2 (Suppl III): 132-8, 1983.

13. Morice $\mathrm{AH}$, Brown MJ, Higenbottam T. Cough associated with angiotensin converting enzyme inhibi- 
tors. J Cardiovasc Pharmacol 1989; 13 (Suppl 3): S59-S62.

14. Frdos EG, Skidgel RA. The unusual substrate specificity and the distribution of human angiotensin I converting enzyme. Hypertension 1986; 8 (Suppl I): 34-7.

15. Sun J, Elwood W, Barnes PJ, Chung KF. Effect of thiazide diuretics against neurally mediated contraction of guinea pig airways. Contribution of carbonic anhydrase. Am Rev Respir Dis 1993; 148: 9028.

16. Karlsson JA, Choudry NB, Zackrisson C, Fuller RW. A comparison of the effect of inhaled diuretics on airway reflexes in humans and guinea pigs. 1992; J Appl Physiol 72: 434-8.

17. McEwan JR, Choudry NB, Fuller RW. The effect of sulindac on the abnormal cough reflex associated with dry cough. J Pharm Exp Ther 1990; 255: 161-4.

18. Hirakata H, Onoyama K, Iseki K, Kumagai H, Fujimi S, Omae T. Worsening of anemia induced by long-term use of captopril in hemodialysis patients. Am J Nephrol 1984; 4: 355-60.

19. Loftus WK, Ierino F, Mathew TH. Enalapril and anemia. Med J Aust 1988; 148; 209-10.

20. Gould AB, Goodman S, DeWolf R, Onesti G, Schwarz C. Interrelation of the renin system and erythropoietin in rats. $\mathrm{J}$ Lab Clin Med 1980; 96: 523-34.

21. Islam MS, Bourbigot B, Codet JP, Songy B, Fournier G, Cledes J. Captopril induces correction of postrenal transplant erythremia. Transplant Int 1990; 3: $222-5$.

22. Paolisso G, Gambardella A, Verza M, D'Amore A, Sgambato S, Varricchio M. ACE inhibition improves insulin-sensitivity in aged insulin-resistant hypertensive patients. J Hum Hypertens 1992; 6: $175-9$.

23. Sasaki J, Arakawa K. Effect of captopril on serum lipids, lipoproteins, and in patients with mild essential hypertension. Curr Ther Res 1986; 40: 898-902.

24. Berglund $\mathrm{G}$, Anderson $\mathrm{O}$. Beta-blockers or diuretics in hypertension?: a six year follow up of blood pressure and metabolic side effects. Lancet 1981; i: 744-7.

25. Gifford RW. The role of diuretics in the treatment of hypertension. Am J Med 1984; 77 (Suppl 4A): $102-6$ 